\title{
Gain of transcription factor binding sites is associated to changes in the expression signature of human brain and testis and is correlated to genes with higher expression breadth
}

\author{
Vandeclécio Lira da Silva ${ }^{1}$, André Mauricio Ribeiro dos Santos ${ }^{1,2}$, Wilfredo Blanco ${ }^{3} \&$ \\ Sandro José de Souza ${ }^{4 *}$ \\ ${ }^{1}$ Bioinformatics Multidisciplinary Environment (BioME), Universidade Federal do Rio Grande do Norte (UFRN), Natal 59078-400, Brazil; \\ ${ }^{2}$ Programa de Pós-Graduação em Genética e Biologia Molecular (PPGBM), Universidade Federal do Pará (UFPA), Belem 66075-110, Brazil; \\ ${ }^{3}$ Department of Computer Science, Universidade Estadual do Rio Grande do Norte (UERN), Natal 59120-450, Brazil; \\ ${ }^{4}$ Brain Institute, Universidade Federal do Rio Grande do Norte (UFRN), Natal 59056-450, Brazil
}

Received September 20, 2018; accepted October 15, 2018; published online March 22, 2019

\begin{abstract}
The gain of transcription factor binding sites (TFBS) is believed to represent one of the major causes of biological innovation. Here we used strategies based on comparative genomics to identify 21,822 TFBS specific to the human lineage (TFBS-HS), when compared to chimpanzee and gorilla genomes. More than $40 \%(9,206)$ of these TFBS-HS are in the vicinity of 1,283 genes. A comparison of the expression pattern of these genes and the corresponding orthologs in chimpanzee and gorilla identified genes differentially expressed in human tissues. These genes show a more divergent expression pattern in the human testis and brain, suggesting a role for positive selection in the fixation of TFBS gains. Genes associated with TFBS-HS were enriched in gene ontology categories related to transcriptional regulation, signaling, differentiation/development and nervous system. Furthermore, genes associated with TFBS-HS present a higher expression breadth when compared to genes in general. This biased distribution is due to a preferential gain of TFBS in genes with higher expression breadth rather than a shift in the expression pattern after the gain of TFBS.
\end{abstract}

TFBS, transcript factor, human evolution, expression breadth

Citation: da Silva, V.L., Santos, A.M.R., Blanco, W., and de Souza, S.J. (2019). Gain of transcription factor binding sites is associated to changes in the expression signature of human brain and testis and is correlated to genes with higher expression breadth. Sci China Life Sci 62, https://doi.org/ $10.1007 / \mathrm{s} 11427-018-9454-7$

\section{INTRODUCTION}

Gene expression in eukaryotes is generally controlled by sequence elements that are binding sites for transcription factors. These transcription factor-binding sites (TFBS) are biased towards a more proximal position regarding the transcription start site (TSS) of genes. Several reports in the last few years have addressed the evolutionary forces acting on TFBS both at inter and intra-species levels (Wray, 2007;

*Corresponding author (email: sandro@neuro.ufrn.br)
Kasowski et al., 2010; Ni et al., 2012; Arbiza et al., 2013; Marnetto et al., 2014; Ribeiro-dos-Santos et al., 2015; Tuğrul et al., 2015). A major conclusion from most of these studies is that the gain/loss of functional TFBS has an important role in evolution through the rewiring of genetic regulatory networks. Several phenotypic traits in metazoa, for example, have evolved through the gain/loss of TFBS (Miller et al., 2007; Rebeiz et al., 2012).

Special interest has been given to the evolution of TFBS in humans. Arbiza et al. (2013), for example, identified many adaptive variations in TFBS, although in general the reg- 
ulatory regions were under weaker selection than protein coding regions. More recently, our group has shown that indels affecting TFBS show a differential frequency in human populations suggesting a role of positive selection in the recent evolution of TFBS in humans (Ribeiro-dos-Santos et al., 2015). In a similar approach as the one described here, Marnetto et al. (2014) has identified human-specific regulatory regions (HSRR) especially in the context of TFBS gain in the human lineage and has associated these regions with ontologies related to the nervous system. This is particularly interesting since some reports have shown that new human genes have evolved functions related to the nervous system (Zhang et al., 2012; Zhang et al., 2015). Here, we further explore the evolution of TFBS in the human lineage, by identifying those binding sites that are only found in humans when compared to chimpanzee and gorilla. Humanspecific regions containing TFBS (TFBS-HS) were identified and we have confirmed that genes associated to these regions are related to the nervous system, among other ontologies. Furthermore, we show that such genes present a trend to be more divergently expressed in human brain and testis. Finally, we show that the gain of TFBS-HS is biased toward genes with high expression breadth. We discuss the implications of such findings on the evolution of TFBS in the human lineage.

\section{RESULTS AND DISCUSSION}

\section{Identification of human-specific sequences in the reference human genome containing transcription factor binding sites}

A region of the reference human genome was considered human-specific if it does not align with the reference sequence from both chimpanzee and gorilla genomes. To identify such regions, we parsed the human/chimpanzee and human/gorilla genome alignments (chain files) available from the UCSC Genome Portal (http://genome.ucsc.edu/) and used liftOver to guarantee the human specificity of the identified regions. Next, the set of 2.69 million TFBS identified by the ENCODE project (Dunham et al., 2012) was used to identify TFBS located within human-specific regions (TFBS-HS). A schematic view of this part of our pipeline is shown in Figure 1B. As shown in Figure 1A, 21,822 TFBSHS were identified. Previous work from our group (Ribeirodos-Santos et al., 2015) has indicated that TFBS located within polymorphic indels in the human genome were biased toward some transcription factors. A similar strategy was applied here to evaluate whether the same bias could be found in the set of TFBS-HS. Table S1 in Supporting Information lists all the transcription factors whose binding sites are biased towards the human-specific genome regions (using a threshold of $P<0.01$ ). Among the TFs enriched in the set of TFBS-HS is ZBTB33, which has been implicated in the regulation of behavior and other features associated to the central nervous system in humans (Kulikov et al., 2016). Another interesting example is THAP1, a TF involved in dystonia (Fuchs et al., 2009).

\section{Differential expression of genes associated to TFBS-HS}

To identify genes associated with TFBS-HS, a $6 \mathrm{~kb}$ window was established flanking all human genes identified by the Reference Sequence Project (see Methods for more details and Figure 1C for a schematic view of this part of our pipeline). The $6 \mathrm{~kb}$ window comprised $5 \mathrm{~kb}$ upstream of the $5^{\prime}$ most transcription start site (TSS) of a given gene plus $1 \mathrm{~kb}$ downstream of the same TSS. The size of the flanking window was based on previous reports from the literature including a recent one by us (Arbiza et al., 2013; Marnetto et al., 2014; Ribeiro-dos-Santos et al., 2015). We found that 9,206 TFBS $(42 \%$ of all 21,822$)$ fall within the $6 \mathrm{~kb}$ window flanking the TSS of 1,283 human genes, reinforcing the notion that TFBS are biased toward the vicinity of TSS. On average, these human genes have 7.17 human-specific TFBS. It is reasonable to speculate that these human-specific TFBS might have an impact on the level and/or pattern of expression of the corresponding genes. To examine this issue, the expression pattern of these 1,283 genes (and their orthologs in chimpanzee and gorilla) was evaluated in several tissues from all three species using RNA-Seq data from the Mammalian Project (Brawand et al., 2011). First, a differential expression analysis was performed to identify genes whose expression was significantly changed in humans compared to chimpanzee and gorillas. Table S2 in Supporting Information lists all genes identified as differentially expressed in humans, when compared to either chimpanzee or gorilla.

Furthermore, we searched for genes associated to TFBSHS that presented a different expression pattern when human, chimpanzee and gorilla tissues were analyzed. To do that, FPKM values for a given gene in a given tissue were converted to a percentage score that corresponds to the proportional level of expression for that gene in that tissue. A $30 \%$ difference was arbitrarily used as a threshold to identify genes whose expression pattern shifted in humans compared to chimpanzee and gorilla. Table 1 lists all 41 selected genes with the respective normalized scores for all tissues.

Next, two distance metrics were used to measure the expression divergence between all three species in each tissue. Two sets of genes were used: all human genes (and their corresponding orthologs) and genes associated with TFBSHS (and their corresponding orthologs). For each gene we calculated the expression difference between human/gorilla and chimpanzee/gorilla. In that way we used the gorilla ex- 


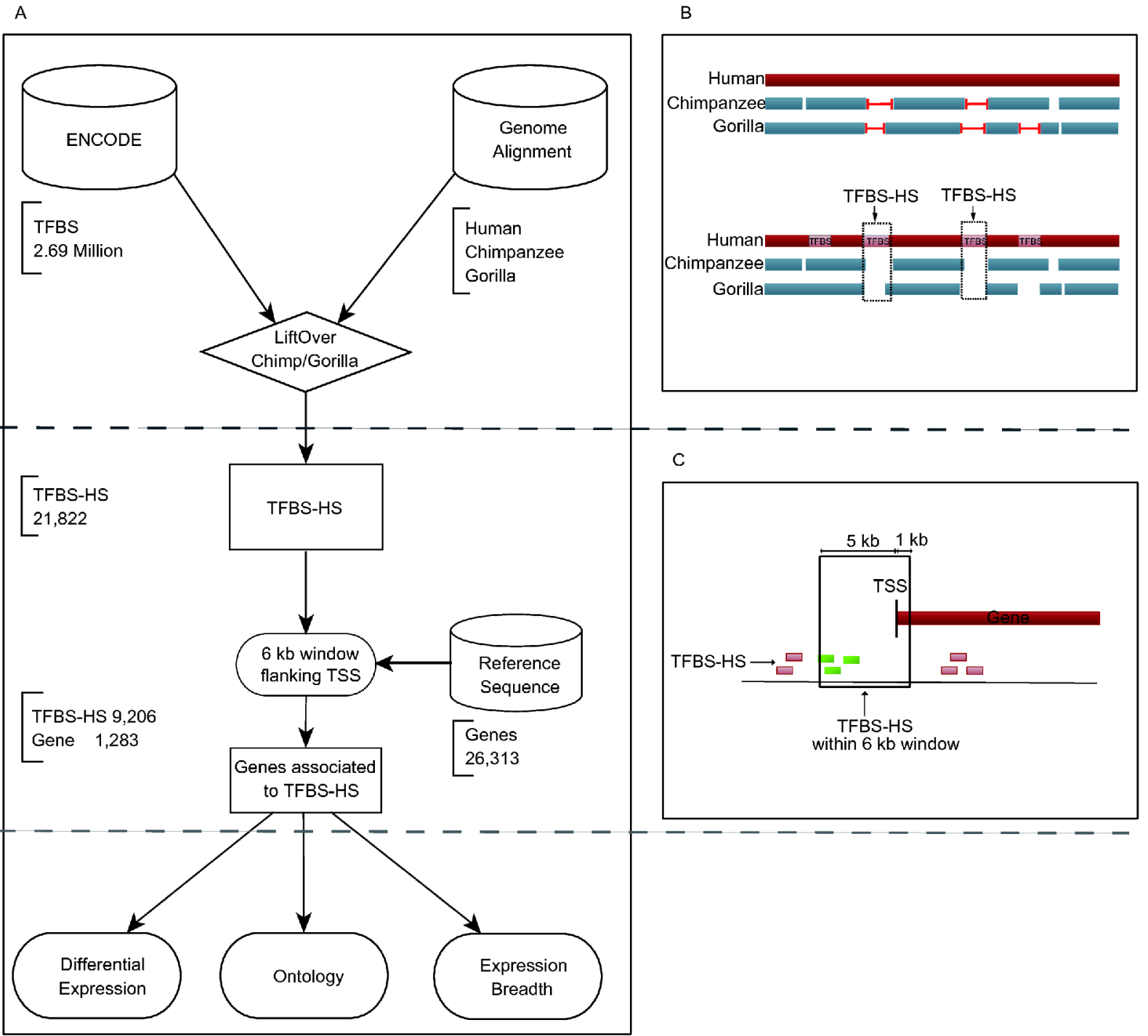

Figure 1 Workflow for the analysis presented here (A). For more details see Methods. Genome coordinates of TFBS mapped to human-specific regions (TFBS-HS) were compared to the coordinates of human Reference Sequences (B). TFBS-HS associated to human genes were defined based on the localization of a given TFBS-HS within a $6 \mathrm{~kb}$ window flanking the TSS (C). The resulting set of 9,206 TFBS and 1,283 genes were then evaluated regarding differential expression, ontology and expression breadth.

pression as a reference and calculated the pace of expression change in human and chimpanzee. Figure 2 shows that gene expression in the brain evolved faster in humans than in chimpanzee when all human genes were used, in accordance with several authors (Enard et al., 2002; Somel et al., 2011). However, we also show that the pace of evolution is even faster in genes associated to TFBS-HS for both brain $(P=0.099)$ and testis $(P=0.001)$. Although the enrichment for the brain is not statistically significant, it shows a clear trend. A second metric was also used to calculate expression divergence in the three primate species. Briefly, expression of a given gene was plotted in a three-axis graph (each axis corresponding to one species). The minimum distance between that datapoint and a hypothetical line representing equal expression in all three species was then calculated.
Again, we used both sets of genes: all human genes and the set of genes associated to TFBS-HS. Table 2 shows the median distance of all orthologous genes in all three species and the set of orthologs to the genes associated to TFBS-HS. Here again, the difference between all human genes and the genes associated to TFBS-HS is more significant for brain and testis, although the difference was statistically significant for all five tissues. This is probably due to the fact that the distance in this second metric reflects expression divergence in all three species.

\section{Gene ontologies enriched in set of genes associated with TFBS-HS}

Although the results presented in Figure 2, Table 1 and Table 
Table 1 Genes differentially represented in human tissues ${ }^{\text {a) }}$

\begin{tabular}{|c|c|c|c|c|}
\hline Gene name & Human $(\%)$ & Chimpanzee (\%) & Gorilla (\%) & Tissues \\
\hline RPS6KA5 & 100.0 & 57.7 & 41.0 & Brain \\
\hline GAREML & 37.3 & 69.3 & 91.8 & Brain \\
\hline PTCHD1 & 58.7 & 94.7 & 96.2 & Brain \\
\hline CAMTA1 & 7.7 & 73.3 & 53.6 & Brain \\
\hline$W N T 7 B$ & 57.3 & 96.2 & 90.9 & Brain \\
\hline$F A M 72 B$ & 100.0 & 13.3 & 21.4 & Brain \\
\hline C17orf67 & 15.5 & 56.2 & 63.6 & Brain \\
\hline FAM228B & 69.3 & 13.8 & 12.3 & Brain \\
\hline SALL3 & 100.0 & 32.3 & 22.4 & Brain \\
\hline STON1-GTF2A1L & 66.7 & 17.9 & 0.1 & Heart \\
\hline C11orf21 & 93.4 & 44.4 & 33.3 & Heart \\
\hline SCUBE3 & 63.2 & 26.2 & 0.0 & Heart \\
\hline$M B 21 D 1$ & 54.2 & 19.6 & 23.5 & Heart \\
\hline$M M P 23 B$ & 100.0 & 55.0 & 58.3 & Heart \\
\hline CXorf40A & 50.0 & 15.6 & 10.8 & Heart \\
\hline$C F D$ & 60.3 & 17.5 & 7.5 & Heart \\
\hline SNORD15B & 41.0 & 0.0 & 0.0 & Heart \\
\hline POLR2J2 & 100.0 & 10.9 & 14.0 & Heart \\
\hline C17orf75 & 88.9 & 10.9 & 18.2 & Kidney \\
\hline$F 2 R L 3$ & 11.0 & 72.7 & 71.4 & Kidney \\
\hline CRIMI & 16.1 & 52.4 & 49.3 & Kidney \\
\hline$I H H$ & 66.9 & 21.6 & 9.0 & Kidney \\
\hline$G B A$ & 0.0 & 62.4 & 40.3 & Kidney \\
\hline$O D F 3 B$ & 4.9 & 44.0 & 35.5 & Kidney \\
\hline$M R G P R X 3$ & 30.3 & 0.0 & 0.0 & Kidney \\
\hline SALL3 & 0.0 & 61.5 & 36.7 & Kidney \\
\hline SYT7 & 32.9 & 0.7 & 1.0 & Liver \\
\hline$R A P 2 C$ & 62.1 & 31.5 & 27.5 & Liver \\
\hline RALGPSI & 36.7 & 0.7 & 2.9 & Liver \\
\hline$M G A T 4 B$ & 83.2 & 28.9 & 32.6 & Liver \\
\hline$I H H$ & 26.8 & 77.7 & 90.5 & Liver \\
\hline CD52 & 2.5 & 37.3 & 53.1 & Liver \\
\hline PUSL1 & 100.0 & 25.0 & 33.3 & Liver \\
\hline$G B A$ & 66.7 & 13.5 & 15.3 & Liver \\
\hline$O D F 3 B$ & 14.7 & 46.6 & 49.1 & Liver \\
\hline$T C F 3$ & 83.4 & 14.1 & 46.9 & Testis \\
\hline SLC35E4 & 63.7 & 6.2 & 17.5 & Testis \\
\hline$T N N C 2$ & 89.9 & 17.5 & 55.3 & Testis \\
\hline KCNN4 & 57.9 & 12.1 & 25.0 & Testis \\
\hline C17orf75 & 0.0 & 40.0 & 48.5 & Testis \\
\hline$V A X 1$ & 61.5 & 9.1 & 0.0 & Testis \\
\hline NOD2 & 33.6 & 2.3 & 0.0 & Testis \\
\hline CD52 & 96.2 & 4.3 & 4.7 & Testis \\
\hline ELP5 & 92.0 & 59.1 & 35.4 & Testis \\
\hline$I R X 2$ & 44.9 & 14.3 & 12.8 & Testis \\
\hline$S H C B P 1$ & 50.3 & 90.2 & 98.4 & Testis \\
\hline$L R R C 37 A$ & 69.0 & 37.6 & 20.3 & Testis \\
\hline$O D F 3 B$ & 44.2 & 3.1 & 5.5 & Testis \\
\hline$M R G P R X 3$ & 36.4 & 80.0 & 100.0 & Testis \\
\hline$F A M 72 B$ & 0.0 & 80.0 & 78.6 & Testis \\
\hline$F A M 228 B$ & 0.0 & 77.3 & 80.2 & Testis \\
\hline
\end{tabular}

a) Values correspond to the proportional level of expression of the corresponding gene in the corresponding tissue. 

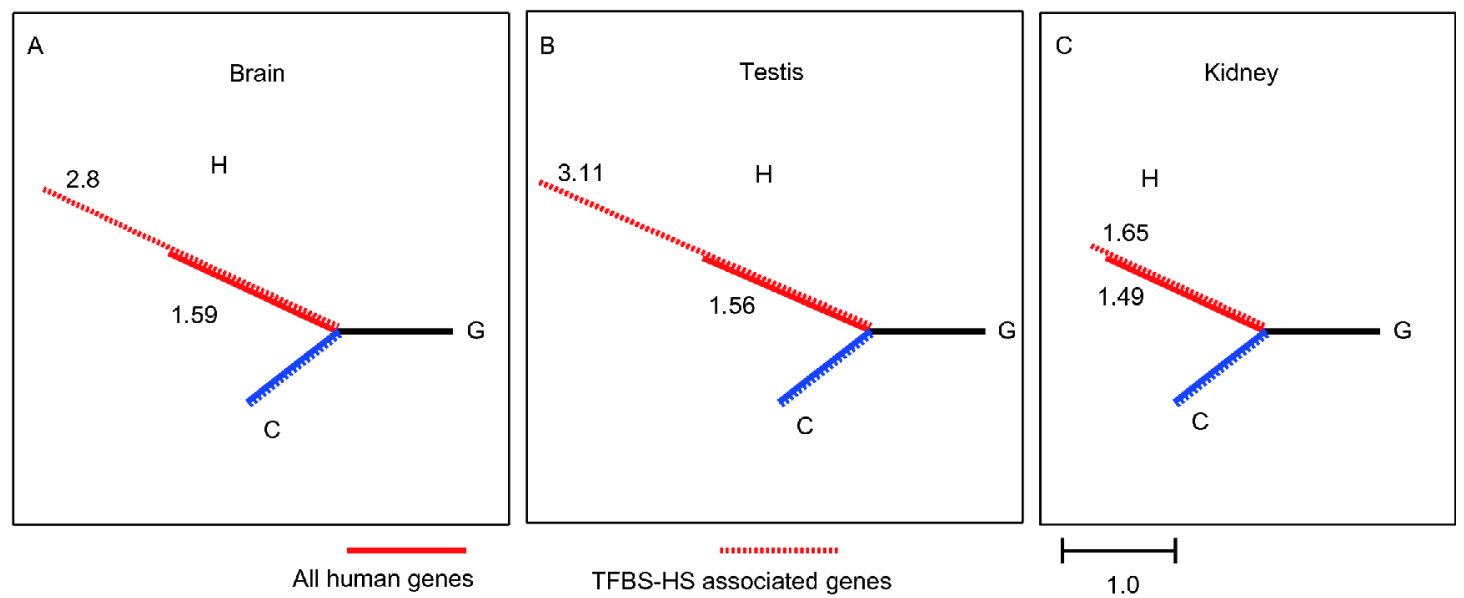

Figure 2 Evolution of gene expression in genes associated to TFBS-HS. Pace of expression change in human and chimpanzee brain (A), testis (B) and kidney (C). Expression of the corresponding genes in gorilla was used as a reference. All human genes are shown in bold line while TFBS-HS associated genes are shown in dashed lines.

Table 2 Expression divergence between species used in this study ${ }^{\text {a) }}$

\begin{tabular}{cccccc}
\hline & Brain & Heart & Liver & Kidney & Testis \\
\hline Median distance (TFBS-HS) & 5.4 & 3.6 & 2.00 & 4.00 & 8.00 \\
Median distance (all orthologous genes) & 2.66 & 2.00 & 1.33 & 3.33 & 5.33 \\
\% increase & 103 & 80 & 50.38 & 20.12 & 50.1 \\
\hline
\end{tabular}

a) Values correspond to the median distance of orthologous genes to a hypothetical line representing equal expression in the same tissue in all three species.

2 are interesting in the sense that they strongly suggest a functional role for TFBS-HS in regulating expression of key genes, many other processes could be responsible for the differential expression of the genes associated to TFBSHS. We decided therefore to evaluate whether any functional category was enriched within the set of genes associated to TFBS-HS. Over representation of functional categories within this set would further argue for an evolutionary role of TFBS-HS in the human lineage. To address this issue, a Gene Ontology enrichment analysis was performed with the 1,283 genes associated to TFBS-HS. Overall, several ontology categories associated to development/differentiation, transcriptional regulation, signal transduction and nervous system were significantly enriched within this gene set (Figure 3). While the same pattern of ontologies has been observed by Marnetto et al. (2014) in their analysis of FHSRR, our enrichment reached higher levels of significance and presented some exclusive ontologies related to transcriptional regulation. It is interesting, however, that two different approaches reached the same overall conclusions reinforcing the notion that human genes with gained TFBS are related to specific biological processes and functions.

\section{Genes associated with TFBS-HS are biased towards high expression breadth}

Motivated by the paper from Hurst et al. (2014), who have developed a metric system able to predict the expression breadth of human genes, we decided to explore whether genes associated with TFBS-HS have a different expression breadth compared to genes overall. First, data from the Illumina Human Body Map was used to compare the expression breadth of all human genes and the 1,283 genes associated with TFBS-HS. As seen in Figure 4A, genes associated with TFBS-HS have a higher expression breadth when compared to all human genes. Two possibilities could account for this biased distribution. First, the presence of human-specific TFBS could change the expression of the respective genes in the human lineage, increasing their expression breadth. Alternatively, the ancestral genes (before the split between human and chimpanzees and the gain of specific TFBS in the human lineage) had already a higher expression breadth and the gains of TFBS occurred predominantly in genes with higher expression breadth. To test these two alternatives, the expression breadth of the chimpanzee and gorilla orthologs of the 1,283 human genes associated to TFBS-HS was evaluated. A higher expression breadth for the orthologs would give support to the second alternative. Otherwise, if the orthologs have a lower expression breadth, the first alternative would be supported. Figure 4C and D clearly show that the orthologs in both chimpanzee and gorilla have an expression breadth higher than the respective total genes. We can then conclude that the gain of TFBS in the human lineage occurred more frequently 


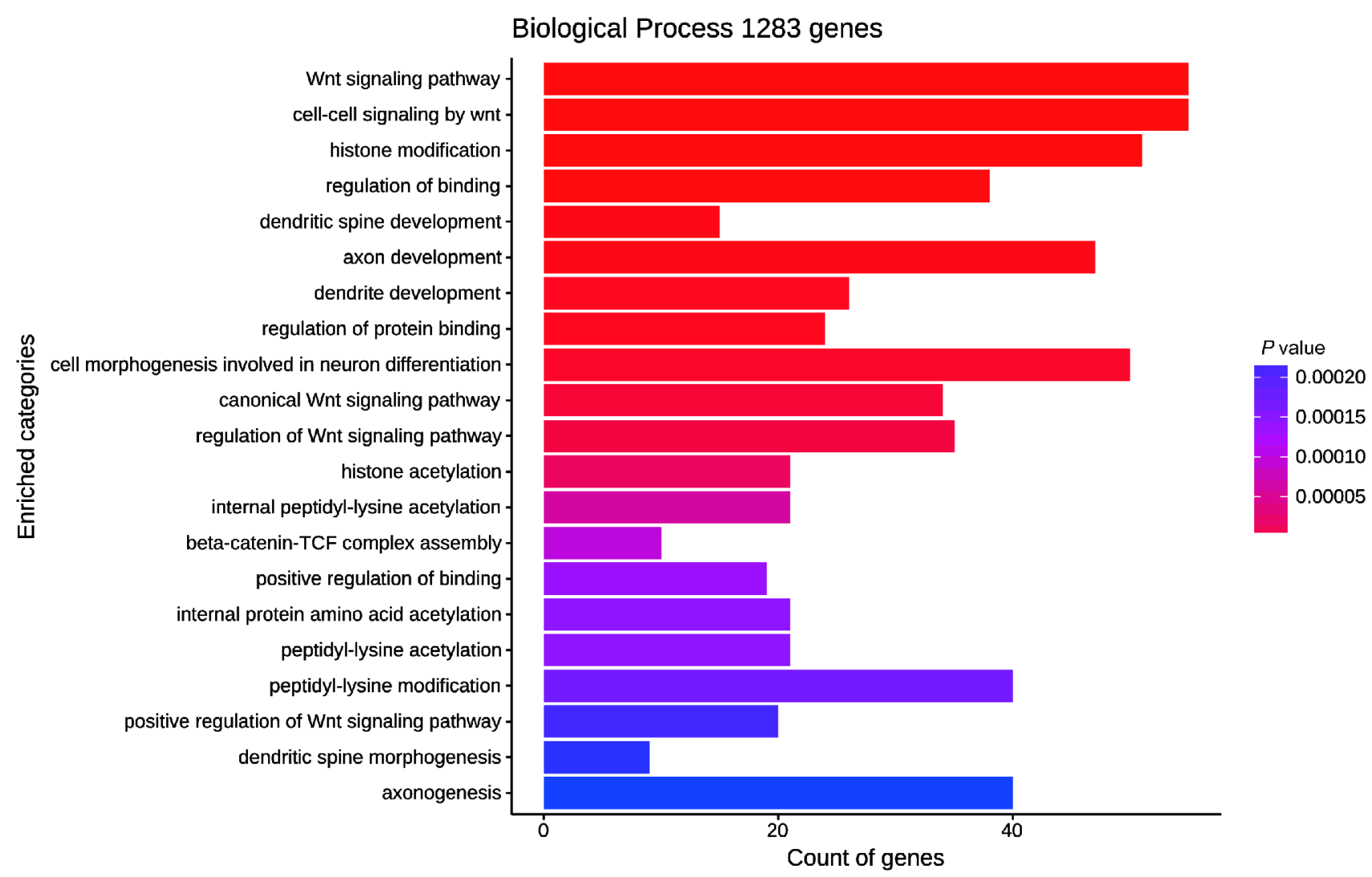

Figure 3 Gene ontology enrichment analysis for human genes associated to TFBS-HS. Bar color refers to the significance of enrichment.

in genes with a higher expression breadth. The reasons behind this biased gain of human-specific TFBS are likely associated to a more relaxed purifying selection in the regulation of transcription in genes with higher expression breadth.

\section{FINAL REMARKS}

We have provided direct evidence that the gain of TFBS in the human lineage is associated with changes in gene expression signatures in the human brain and testis. This suggests that these gains have been under selection in the human lineage. Supporting this view, genes associated with TFBSHS are enriched in ontologies linked to the nervous system. On the other hand, we have also shown that the gain of TFBS is biased towards genes with higher expression breadth, likely due to a more relaxed purifying selection on the regulation of expression of such genes. These apparent contradictory findings can be understood as a dynamic balance of different evolutionary forces acting on the control of gene expression. A complete understanding of such forces will certainly provide insights on the association of eQTLs and several human features, including diseases.

\section{MATERIALS AND METHODS}

\section{Genome alignments}

The following data was obtained from the UCSC Genome Portal (Rosenbloom et al., 2015): (i) Alignments of the human genome against both the chimpanzee and gorilla genomes, (ii) Reference Sequence transcripts and their respective genome alignments, and (iii) TFBS coordinates identified by the ENCODE project V2 (Dunham et al., 2012). Expression data was downloaded from the Mammalian Project (Brawand et al., 2011) and from the Illumina Human Body Map 2.0, both available from the Expression Atlas portal (Petryszak et al., 2014). Information on ortho$\operatorname{logs}$ in all three species was obtained from Ensemble (Cunningham et al., 2015), using the Biomart tool. Orthologous genes were selected based on the best identity and all annotations and analyses were based on the hg19/GRCh37 release of the human genome.

\section{Identification of TFBS-HS}

The genome alignments obtained from UCSC Genome Portal were available in a ".chain" file extension, which has information about the alignment coordinates, the number of matches and the number/position of gaps. To identify se- 

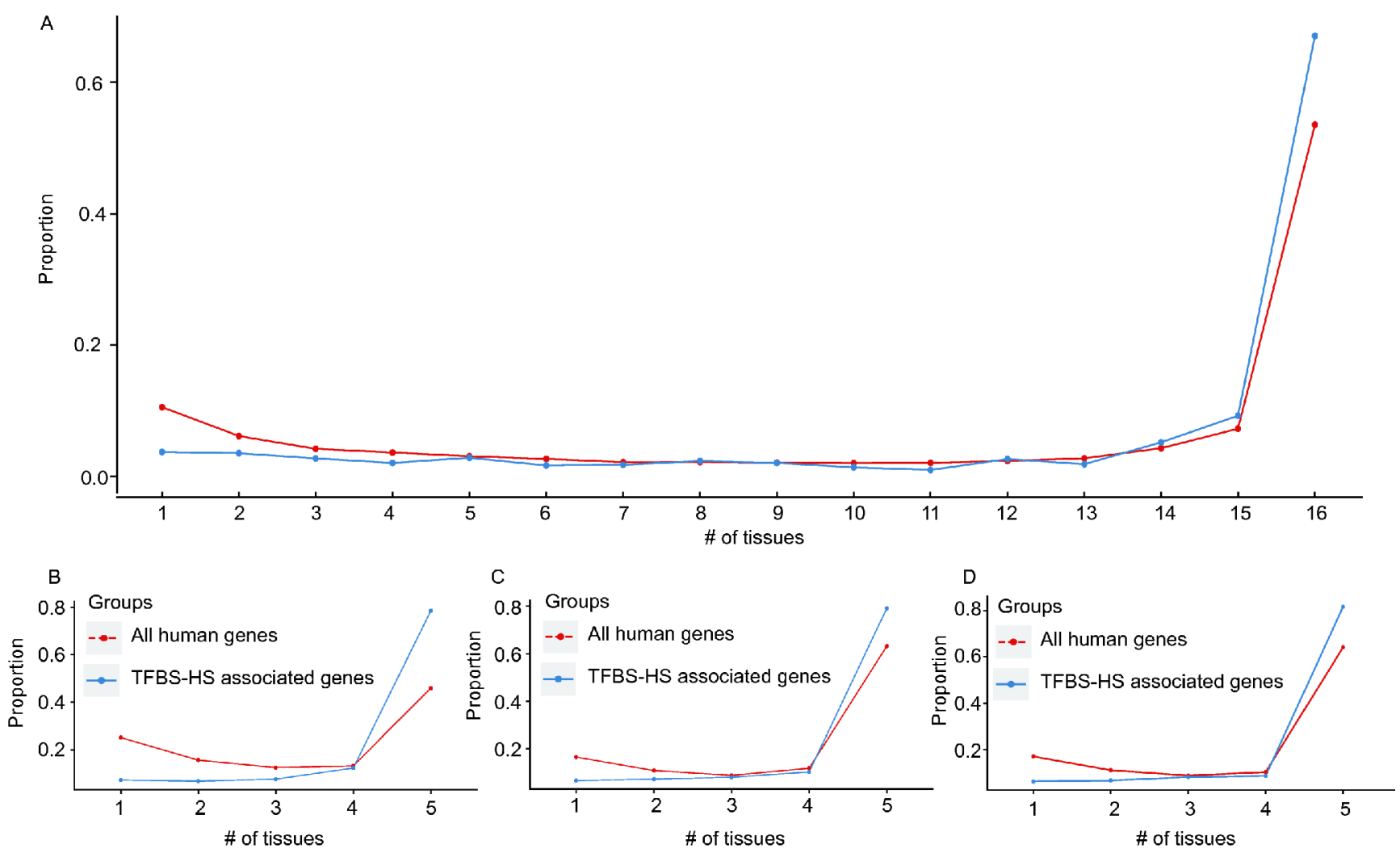

Figure 4 Expression breadth of genes associated to TFBS-HS. A, Pattern of expression breadth for all human genes (red line) and genes associated to TFBS-HS (blue line) using data from the Human Body Map. B, Same analysis as in (A) using data from the Mammalian Project. C, Pattern of expression breadth for all chimpanzee genes (red line) and for the genes orthologous to the human genes associated to TFBS (blue line). D, Pattern of expression breadth for all gorilla genes (red line) and for the genes orthologous to the human genes associated to TFBS (blue line).

quences present only in the human genome, we first selected the most conserved chains from the alignments and then counted the number of matches and gaps by walking on the selected chain. When a gap was larger than 20 bp within the chimpanzee or gorilla genomes, its respective coordinate was compared to the coordinates of all TFBS from ENCODE as shown in Figure 1B. A final step in our pipeline involved the use of liftOver (Kent et al., 2010) in a comparison between our putative human-specific regions to the chimpanzee and gorilla genome sequences. TFBSs co-localizing with humanspecific regions, confirmed by liftOver, were selected and then stored into a MySQL database (Widenius et al., 2002).

\section{Selection of genes in the vicinity of TFBS-HS}

We first selected the largest transcript of each gene from the respective Reference Sequence entry and defined the TSS (Transcription Start Site) for that entry. A $6 \mathrm{~kb}$ region flanking the TSS ( $5 \mathrm{~kb}$ upstream plus $1 \mathrm{~kb}$ downstream of the TSS) was defined and compared to coordinates of all TFBSHS, as shown in Figure 1C. BedTools software (Quinlan and Hall, 2010) was used to identify the intersection between the TFBS-HS and the $6 \mathrm{~kb}$ window.

\section{TFs enrichment analysis}

The enrichment analysis of TFs within the dataset of TFBSHS was performed through a Monte Carlo simulation, where random sets of 9,206 TFBS (same number as TFBS-HS) were tested against all available transcription factors present within the $6 \mathrm{~kb}$ region flanking the TSS of all human genes.

\section{Identification of genes showing differential expression}

The first approach was the direct comparison of FPKM values between the same tissues in different species. First, orthologs genes in the three species were selected and gene expression ratio was calculated between human/chimpanzee and human/gorilla. A threshold was used to select differentially expressed human genes $\left(\log _{2}(\right.$ ratio $)>2$ or $\left.<-2\right)$.

In a second approach, gene expression was normalized by the proportion of each gene expression in each tissue using the data from the Mammalian Project. For each tissue, that was done by taking the expression of a given gene in that tissue and dividing that value by the sum of that gene expression in all tissues. Next, all orthologs were identified (by using the Ensembl database) and differences $>0.3$ for the 
same gene and tissue in different species were selected.

\section{Rate and distance of gene expression change}

To calculate the expression divergence between human genes associated with TFBS-HS and their orthologs in chimpanzee and gorilla, two approaches were used. First, expression from the same tissue in each species was plotted in a three-dimensional graph and the minimum distance between each point and a hypothetical line representing equal expression in all three species was determined. The median of all minimum distances represents a metric for expression divergence. Second, for each pair of orthologs (human/gorilla and chimpanzee/gorilla), we calculated the expression difference between human/gorilla and chimpanzee/gorilla. In that way, the expression in gorilla (outgroup) was used as a reference to calculate the pace of expression change in chimpanzee and human.

\section{Enrichment ontology analysis}

The package of R package 'clusterProfiler version 3.0' was used to perform the enrichment analysis of ontology. The enrichment analysis of both Gene Ontology (GO) and Kyoto Encyclopedia of Genes and Genomes (KEGG) with either hypergeometric test or Gene Set Enrichment Analysis (GSEA) were also performed using clusterProfiler, which adjusts the estimated significance level to account for multiple hypothesis testing (Yu et al., 2012).

\section{Expression breadth analysis}

In-house R scripts (R Core Team, 2013) were developed for the expression breadth analyses based on the data from the Human Body Map Project. Only genes showing official HUGO (Gene Nomenclature Committee, HGNC) were taken into account. Genes were grouped by the number of tissues in which they are expressed. Due to the high sensitivity of RNA-Seq methods, $30 \%$ of the least expressed genes for each tissue were removed. Statistical chi-square tests were performed to check if our set of genes was enriched with genes showing a higher expression breadth.

Compliance and ethics The author(s) declare that they have no conflict of interest.

Acknowledgements The authors are indebted to Jorge E.S. de Souza for discussions on the gene expression analysis. VLS and AMRS were supported by CAPES Ph.D. fellowships. This work was supported by the Ludwig Institute for Cancer Research and by CAPES (23038.004629/2014-19).

\section{References}

Arbiza, L., Gronau, I., Aksoy, B.A., Hubisz, M.J., Gulko, B., Keinan, A., and Siepel, A. (2013). Genome-wide inference of natural selection on human transcription factor binding sites. Nat Genet 45, 723-729.

Brawand, D., Soumillon, M., Necsulea, A., Julien, P., Csárdi, G., Harrigan, P., Weier, M., Liechti, A., Aximu-Petri, A., Kircher, M., et al. (2011). The evolution of gene expression levels in mammalian organs. Nature 478, 343-348.

Cunningham, F., Amode, M.R., Barrell, D., Beal, K., Billis, K., Brent, S., Carvalho-Silva, D., Clapham, P., Coates, G., Fitzgerald, S., et al. (2015). Ensembl 2015. Nucleic Acids Res 43, D662-D669.

Dunham, I., Aldred, S.F., Collins, P.J., Davis, C.A., Doyle, F., Epstein, C. B., Frietze, S., Harrow, J., and Kaul, R. (2012). An integrated encyclopedia of DNA elements in the human genome. Nature 489, 57-74.

Enard, W., Khaitovich, P., Klose, J., Zöllner, S., Heissig, F., Giavalisco, P., Nieselt-Struwe, K., Muchmore, E., Varki, A., Ravid, R., et al. (2002). Intra- and interspecific variation in primate gene expression patterns. Science 296, 340-343.

Fuchs, T., Gavarini, S., Saunders-Pullman, R., Raymond, D., Ehrlich, M.E., Bressman, S.B., and Ozelius, L.J. (2009). Mutations in the THAP1 gene are responsible for DYT6 primary torsion dystonia. Nat Genet $41,286-$ 288.

Hurst, L.D., Sachenkova, O., Daub, C., Forrest, A.R.R., Huminiecki, L., and Huminiecki, L. (2014). A simple metric of promoter architecture robustly predicts expression breadth of human genes suggesting that most transcription factors are positive regulators. Genome Biol 15, 413.

Kasowski, M., Grubert, F., Heffelfinger, C., Hariharan, M., Asabere, A., Waszak, S.M., Habegger, L., Rozowsky, J., Shi, M., Urban, A.E., et al. (2010). Variation in transcription factor binding among humans. Science 328, 232-235.

Kent, W.J., Zweig, A.S., Barber, G., Hinrichs, A.S., and Karolchik, D. (2010). BigWig and BigBed: enabling browsing of large distributed datasets. Bioinformatics 26, 2204-2207.

Kulikov, A.V., Korostina, V.S., Kulikova, E.A., Fursenko, D.V., Akulov, A. E., Moshkin, M.P., and Prokhortchouk, E.B. (2016). Knockout Zbtb33 gene results in an increased locomotion, exploration and pre-pulse inhibition in mice. Behav Brain Res 297, 76-83.

Marnetto, D., Molineris, I., Grassi, E., and Provero, P. (2014). Genomewide identification and characterization of fixed human-specific regulatory regions. Am J Hum Genet 95, 39-48.

Miller, W., Rosenbloom, K., Hardison, R.C., Hou, M., Taylor, J., Raney, B., Burhans, R., King, D.C., Baertsch, R., Blankenberg, D., et al. (2007). 28-Way vertebrate alignment and conservation track in the UCSC Genome Browser. Genome Res 17, 1797-1808.

Ni, X., Zhang, Y.E., Nègre, N., Chen, S., Long, M., and White, K.P. (2012). Adaptive evolution and the birth of CTCF binding sites in the Drosophila genome. PLoS Biol 10, e1001420.

Petryszak, R., Burdett, T., Fiorelli, B., Fonseca, N.A., Gonzalez-Porta, M., Hastings, E., Huber, W., Jupp, S., Keays, M., Kryvych, N., et al. (2014). Expression Atlas update - a database of gene and transcript expression from microarray- and sequencing-based functional genomics experiments. Nucl Acids Res 42, D926-D932.

Quinlan, A.R., and Hall, I.M. (2010). BEDTools: A flexible suite of utilities for comparing genomic features. Bioinformatics 26, 841-842.

R Core Team. (2013). R: A language and environment for statistical computing. doi: 10.1007/978-3-540-74686-7.

Rebeiz, M., Castro, B., Liu, F., Yue, F., and Posakony, J.W. (2012). Ancestral and conserved cis-regulatory architectures in developmental control genes. Dev Biol 362, 282-294.

Ribeiro-dos-Santos, A.M., da Silva, V.L., de Souza, J.E.S., and de Souza, S. J. (2015). Populational landscape of INDELs affecting transcription factor-binding sites in humans. BMC Genom 16, 536.

Rosenbloom, K.R., Armstrong, J., Barber, G.P., Casper, J., Clawson, H., Diekhans, M., Dreszer, T.R., Fujita, P.A., Guruvadoo, L., Haeussler, M., et al. (2015). The UCSC Genome Browser database: 2015 update. Nucleic Acids Res 43, D670-D681.

Somel, M., Liu, X., Tang, L., Yan, Z., Hu, H., Guo, S., Jiang, X., Zhang, X., Xu, G., Xie, G., et al. (2011). MicroRNA-driven developmental 
remodeling in the brain distinguishes humans from other primates. PLoS Biol 9, e1001214.

Tuğrul, M., Paixão, T., Barton, N.H., and Tkačik, G. (2015). Dynamics of transcription factor binding site evolution. PLoS Genet 11, e1005639.

Widenius, M., Axmark, D., and DuBois, P. (2002). MySQL reference manual: documentation from the source (Beijing: O'Reilly, Community Press).

Wray, G.A. (2007). The evolutionary significance of cis-regulatory mutations. Nat Rev Genet 8, 206-216.
Yu, G., Wang, L.G., Han, Y., and He, Q.Y. (2012). clusterProfiler: an R package for comparing biological themes among gene clusters. OMICS $16,284-287$.

Zhang, W., Landback, P., Gschwend, A.R., Shen, B., and Long, M. (2015). New genes drive the evolution of gene interaction networks in the human and mouse genomes. Genome Biol 16, 202.

Zhang, Y.E., Landback, P., Vibranovski, M., and Long, M. (2012). New genes expressed in human brains: implications for annotating evolving genomes. Bioessays 34, 982-991.

\section{SUPPORTING INFORMATION}

Table S1 Enrichment analysis of transcription factors within the set of TFBS-HS

Table S2 Differential expression of genes associated to TFBS-HS

The supporting information is available online at http://life.scichina.com and http://link.springer.com. The supporting materials are published as submitted, without typesetting or editing. The responsibility for scientific accuracy and content remains entirely with the authors. 